\title{
Mathematical Decision Model for Reverse Supply Chains Inventory
}

\author{
L. Duta, C.B. Zamfirescu, F.G. Filip
}

\author{
Luminita Duta \\ Automation and Computer Science Department \\ Valahia University of Targoviste \\ Romania, 130083, Targoviste, 24, Unirii Ave. \\ duta@valahia.ro

\section{Constantin-Bala Zamfirescu} \\ Dept. of Computer Science and Automatic Control \\ Lucian Blaga University of Sibiu \\ Romania, 550024, Sibiu, 10, Victoriei Ave. \\ zbc@acm.org

\section{Florin G. Filip} \\ The Romanian Academy \\ Romania, Bucharest, 010071, 125 Calea Victoriei \\ ffilip@acad.ro
}

\begin{abstract}
In the reverse supply chain inventory theory, inventory models are concerned with the demand of reusable parts, stock replenishment, ordering cycle, delivery lead time, number of disassembled products, ordering costs. The particularity of these models consists in the occurrence of high uncertainties of the quantity and quality of the returned products and resulting parts. To overcome the problem, an inventory model that incorporates decision variables at proactive and reactive levels is derived and discussed in this paper.
\end{abstract}

Keywords: Reverse supply chains, decision aid, inventory models, Bayesian networks

\section{Introduction}

A reverse supply chain is the process of moving End of Life (EOL) products from customer to distributor or manufacturer for the purpose of capturing the market leftover value, or for proper disposal. Reverse supply chains operations have a strong impact on forward supply chain stocks and transportation capacities. Customers act as suppliers in reverse supply chains. After sorting, the collected items will be moved through the reverse supply chain. Some used products are sold for recycling, usually after destroying their physical structure. Others are used as source of spare parts on the secondary market. Another common use of returns is as spare parts for warranty claims in order to reduce the cost of providing these services to customers. Defective and used products are moved to manufacturer for reusing or remanufacturing or for disposal. Remanufactured or refurbished products are sold for additional revenue to a secondary market.

Part of the reverse supply chain, the reverse logistics has to deal with product transportation, production planning and inventory management. In reverse supply chain inventory, two problems might occur: a) a high level of uncertainty provided by the product end-of-life state of the EOL product and b) the irregular supply for remanufacturing or refurbishing. In this respect, two types of inventory replenishment models have been identified in literature: a deterministic model with stationary or dynamical demand and a stochastic model with continuous or periodical review [1]. This paper addresses the stochastic models with variability of the demand. 
In the sequel the paper is organized as it follows. The state of art from the second section of this paper describes various reverse supply chain inventory models found in literature. In the third section we present a brief review of supply chain inventory policies and the positioning of our work. In the fourth section, an approach based on a mathematical decision model to deal with the stochastic aspect of the reverse supply chain inventory is described. Decision variables are introduced on two levels: a proactive level and a reactive level. Results and discussions are highlighted in the fifth section. Conclusions and future work perspectives are presented in the last part of the paper.

\section{Literature review}

Early researches in the domain of interest of this paper concerned the economic order quantity logic which was exploited by using deterministic models with stationary demand. In [2] a survey on quantitative models for reverse logistics is given. In such systems, authors show that the objective of the inventory management is to control the component recovery process to guarantee a required level of maintenance service and to minimize fixed and variable costs.

In [3] Kiesmuller and Sherer determinate, for the first time, an optimal policy for a remanufacturing system with dynamic demand, by assuming that there are no backorders and lead times. In [4], authors develop two heuristic procedures to investigate the effect of stochastic yields on a disassembly to order system. In [5], Imtavanich and Gupta use heuristics to address different stochastic parameters of a disassembly to order (DTO) system and they propose a goal programming procedure to calculate the number of returned products that satisfy various goals. A DTO system is concerned with the process of finding how many products have to be disassembled in order to fulfil the demand of reusable parts or subassemblies that will be used in remanufacturing. Bayindira and et al. [6] investigate the desired level of recovery under various inventory control policies when the success of recovery is probabilistic. All used and returned items go into a recovery process that is modelled as a single stage operation. The recovery effort is represented by the expected time spent for it. Using brand new parts may represent an alternative or/and complementary solution to ensure the necessary stocks for satisfying the orders received in case the recovered parts do not satisfy the demand. Four inventory control policies that differ in timing of and information used in purchasing decision could be envisaged. The objective is to find the recovery level together with inventory control parameter that minimizes the long-run average total cost. A numerical study covering a wide range of system parameters is carried out. Finally computational results are presented with their managerial implications. A DTO model with deterministic yields is presented in [7]. The DTO system is represented by an integer programming model. The objective function is to minimize the total costs. The authors propose three decision variables: one $v_{1}$ for the amount of the end of life EOL products to be acquired in view of disassembling, one $v_{2}$ for the amount of new parts to be acquired in order to meet the overall demand and one $v_{3}$ for the number of the parts that need to be disposed.

The authors made several assumptions: there is no lead time for acquisition or disassembly, products are completely disassembled, there is a continuous supply of EOL products and the demand for parts is completely fulfilled. In [8], a decisional DTO decision model is presented. The author separated decomposes the decision process into two stages. In the first stage one the goal is to minimize the costs by deciding how many items must be disassembled to fulfil the reusable parts demand. In the second stage the goal is to minimise the number of disposed parts and the procurement costs of the new parts. Another model for DTO systems with stochastic yields is presented by Kongar and Gupta in [9]. The quantity of reusable parts is obtained from the number of EOL products disassembled multiply by a weight coefficient. The method uses models in which linear programming encompasses a degree of stochasticity. Decisions are split 
into two groups: proactive decisions and reactive decisions. While proactive decisions determine the number of EOL products to disassemble, reactive decisions take into account the uncertain variables (as the quality of the disassembled part, the lead time or the disassembly time) and their probability of occurrence. Kongar and Ilgin propose a linear physical programming solution based methodology which deals with tangible and intangible financial, environmental and performance measures of DTO systems [10]. Two types of decision depending on the disassembly operations are taken into consideration. If the item (the EOL product) is subjected to resale or storage, a non-destructive disassembly process is performed. Otherwise, if the item is subjected to recycling or disposal, a destructive disassembly is applied [9]. In 2010, Ahiska and King [11] extend Kiesmullerand Sheres' approach [3] by considering different lead times for manufacturing and remanufacturing. Recently, Godichaud [12] demonstrates the relevance of Bayesian Networks $(\mathrm{BN})$ in a spare parts inventory model which deals with costs and time uncertainties. The same tool (BN) is used in [13] to highlight the temporal dependences between variables in a model for optimal disassembly policy.

\section{Inventory policies for reverse supply chain}

The economic order quantity model (EOQ) is one of the most widely known inventory control methods [14]. It is used to set the quantities to order in replenishing inventories so that a trade-off between inventory holdings and ordering/set up costs is achieved. Some assumptions are associated with this model: a) constant and known demand, instantaneous receipt of inventory, b) constant and known time intervals between order placement and receipt of the order, stock outs are avoided by placing orders at the right time. It is also assumed that the unit procurement price remains constant irrespective of the number of units purchased. This model induces four inventory basic policies according to the moment of the inventory position review: with continuous review or with periodical review. Used notations: $\mathrm{Q}=$ order quantity, $\mathrm{S}=$ order-up-to level, $\mathrm{s}=$ reorder point, $\mathrm{T}=$ review period [15]:

- The $(s, Q)$ Policy: Whenever the inventory position (inventory level plus quantity on order) drops to a given level limit, $s$, or below, an order is placed for a fixed quantity, $Q$.

- The $(s, S)$ Policy: Whenever the inventory position (inventory level plus quantity on order) drops to a given level $s$, or below, an order is placed for a sufficient quantity to bring the inventory position up to a given level, $S$.

- The $(T, S)$ Policy: Inventory position is reviewed at regular discrete time moments spaced at intervals of length $T$ time units. At each review, an order is placed for a sufficient quantity to bring the inventory position up to a given level, $S$.

- The $(T, s, S)$ Policy: Inventory position is reviewed at regular instants spaced at time intervals of length T. At each review, if the inventory position is at level $s$ or below, an order is placed for a sufficient quantity to bring the inventory position up to a given level $S$. If the inventory position is above s, no order is placed.

Remarks:

- When $T=0$ in the $(T, s, S)$ policy, one obtains the $(s, S)$ policy. So, the $(T, s, S)$ policy can be regarded as a periodic version of the $(s, S)$ policy, which, in turn, may be viewed as a special case of the $(T, s, S)$.

- The $(T, S)$ policy represents a special case of the $(T, s, S)$ policy in which $\mathrm{s}=\mathrm{S}$ 


\section{The decision model}

Since the demand for spare parts is variable and is depending on the needs and the time when an order for replenishment is placed until the replenishment arrives (the lead time), the $(\mathrm{T}, \mathrm{s}, \mathrm{S})$ policy was considered in this paper. In such a system, the period of review is fixed and the ordered quantity changes as per demand or rate of consumption. The period of review $\mathrm{T}$ is decided such as the ordered quantity is economical for purchasing the items. The problem is to coordinate the two processes - disassembly and remanufacturing - so as to meet the demand of items. Another problem is the disassembly depth that deals with how completely a product should be disassembled. In this context, the researcher must weigh not only the costs of the process, whether is destructive or not, but also consider which reusable parts are already in stock, how many will be obtained through disassembly and will be accumulated in inventory and how many parts will have to be disposed of. It is obvious that a reverse chain inventory model has to include different decision variables.

\subsection{Notations}

$N_{i}^{d}=$ Number of items $i$ to acquire and disassemble

$P_{k i}=$ Amount of part $k$ in item $i$

$P_{k i}^{r}=$ Amount of part $k$ in item $i$ to reuse

$P_{k i}^{d}=$ Amount of part $k$ in item $i$ to dispose

$P_{k i}^{h}=$ Amount of part $k$ in item $i$ to hold (to stock)

$C_{i}^{a}=$ Acquisition cost of the item $i$

$C_{i}^{d}=$ Disassemble cost of the item $i$

$C_{k}^{r}=$ Reusing cost of the part $k$ of the item $i$

$C_{k}^{d}=$ Disposal cost of the part $k$ of the item $i$

$C_{k}^{h}=$ Holding cost of the part $k$ of the item $i$

$P_{s c}=$ Probability of scenario $s c$ occurring

$T C=$ total inventory cost

To simplify the analytical model, the following assumptions are made:

- Only a single type of product to disassembly is considered;

- There is no lead times for acquisition or disassembly;

- EOL items to disassembly are always available;

- A single disassembly scenario $\left(P_{s c}=1\right)$ is occurring;

- Products are completely disassembled;

- There are only two types of EOL options: reusing and disposal;

- Two types of disassembly operations are considered: destructive and non-destructive;

- All costs are deterministic and constant;

- The interval to acquire is deterministic (at the first slice of time $t$ );

- The amount of reusable, disposable and holding parts is subjected to uncertainties;

- The number of items to acquire at the second slice of time have a probabilistic distribution;

- The model is periodically reviewed [8]. 


\subsection{The Bayesian network model}

To implement the correct model, Bayesian Networks were used so as to determinate all influences and causalities between decision variables. The results will show how information influences decisions and how these decisions cause the change of information.

Bayesian networks (BN) have the ability of capturing both qualitative knowledge through their network structure, and quantitative knowledge through their parameters [14]. A static Bayesian Network can be extended to a Dynamic Bayesian Network (DBN) by introducing relevant temporal dependencies to capture the dynamic behaviors of the system at different moments.

To validate the model, the BayesiaLab@ software is used ( [16] and [17]). The software is able to seize degrees of probability. Once validated, probabilities are used jointly with the probability distribution for giving a new Probability distribution. BayesiaLabß allows the temporal dimension integration in a Bayesian Network. Thus, a BN can be easily transformed into a DBN. Temporal nodes at instants $t$ and $t+1$ can be represented and connected by temporal arcs. The parameters evolution of the DBN nodes can be so tracked in time.Decision nodes are marked by squares. The amount of part $k$ found in item $i$ influences the amount to reuse, to dispose or to hold. Further, these decisional variables change the number of products to be acquired at the next slice of time (moment $t+1$ ). The two temporal nodes $N i d(t)$ and $N i d(t+1)$ are linked by a temporal arc. Figure 1 shows background calculation of the total cost. The objective function is included in the utility node TC. In the figure, two decision nodes are represented: Da - decision to acquire used products, Dd - decision to disassembly.

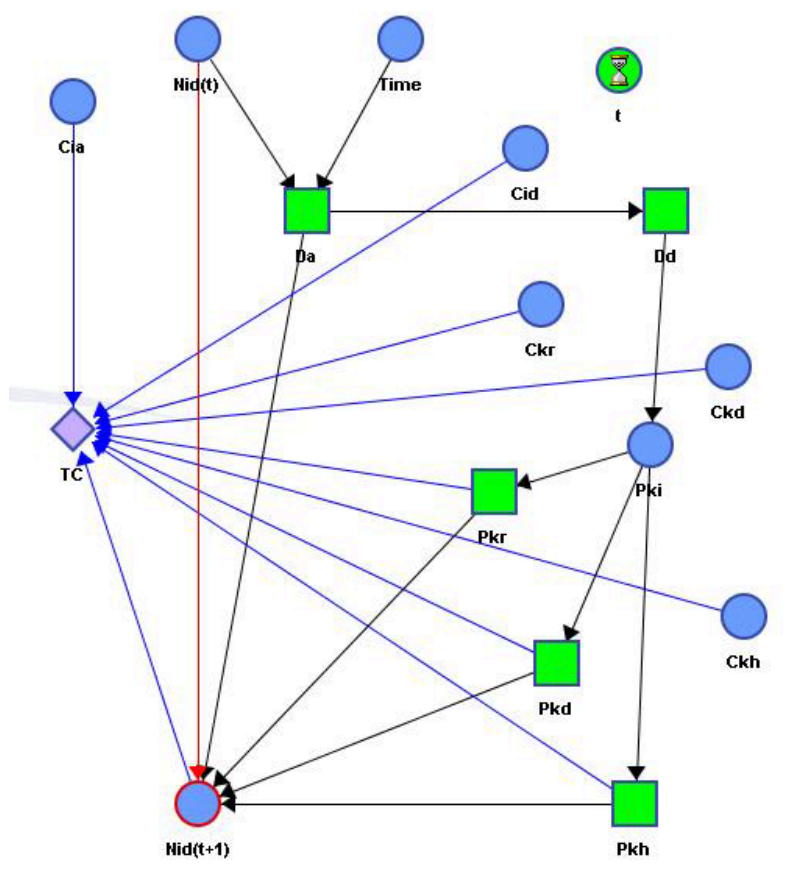

Figure 1: Dynamic Bayesian Network Model

\subsection{The mathematical model}

The total cost of the disassembly phase (TC) is composed of product acquisition cost, disassembly operations costs, EOL options costs and inventory cost. The value of the total cost is given by the equation (1). The first term is the cost of product acquisition and disassembly so 
as to meet the demand of parts for remanufacturing, while the second term is the inventory cost of the disassembled parts. This function is a multi-objective one, since the aim is to find not only the optimal quantity of products to acquire to meet the demand of parts, but also to find the best EOL strategy or scenario so as to optimize the number of parts to reuse in a remanufactured product. For the moment we are interested on quantity to acquire to meet the demand, while scenarios probabilities are introduced by the software to reduce the objective function to a mono-objective one.

The objective function to be minimized is:

$$
\operatorname{Min}(T C)=\operatorname{Min}\left(\sum_{i}\left(C_{i}^{a}+C_{i}^{d}\right) \cdot N_{i}^{d}+\sum_{i} \sum_{s c} P_{s c}\left(C_{k}^{r} \cdot P_{k i}^{r}+C_{k}^{d} \cdot P_{k i}^{d}+C_{k}^{h} \cdot P_{k i}^{h}\right)\right)
$$

In equation 1 the unknown variables are $N_{i}^{d}, P_{k}^{r}, P_{k}^{d}, P_{k}^{h}$.

This objective function is subjected to the following constraints:

$$
\begin{gathered}
d_{k} \leqslant \sum_{i} \sum_{s c} P_{s c} \cdot\left(P_{k i}-P_{k i}^{d}\right) \\
N_{i}^{d} \geq 0 \text { and integer } \\
P_{k i}, P_{k}^{r}, P_{k}^{d}, P_{k}^{h} \geq 0 \text { and integer } \\
\sum_{s c} P_{s c}=1
\end{gathered}
$$

Where $d_{k}$ is the demand (the number of $k$ parts needed). One can note that equations (1) to (5) form a linear integer mathematical model where decision variables can be treated as continuous in order to satisfy the integer value of the demand.

\section{Results}

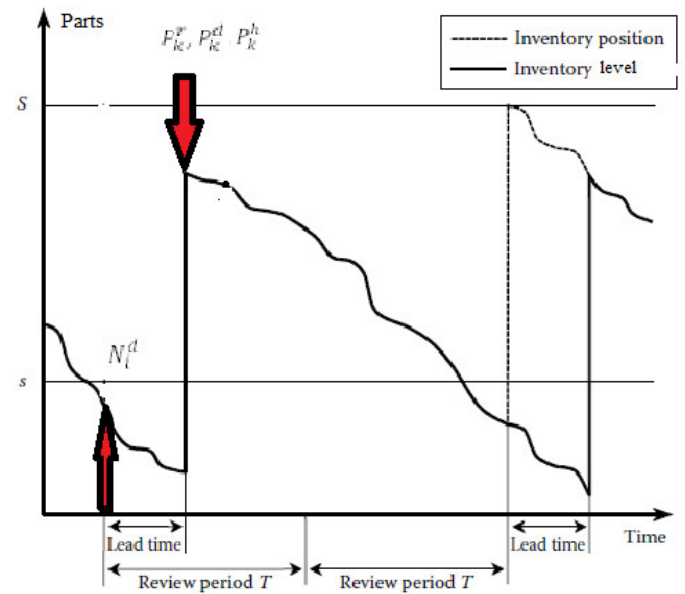

Figure 2: $(T, s, S)$ policy decisions( [13], p 117)

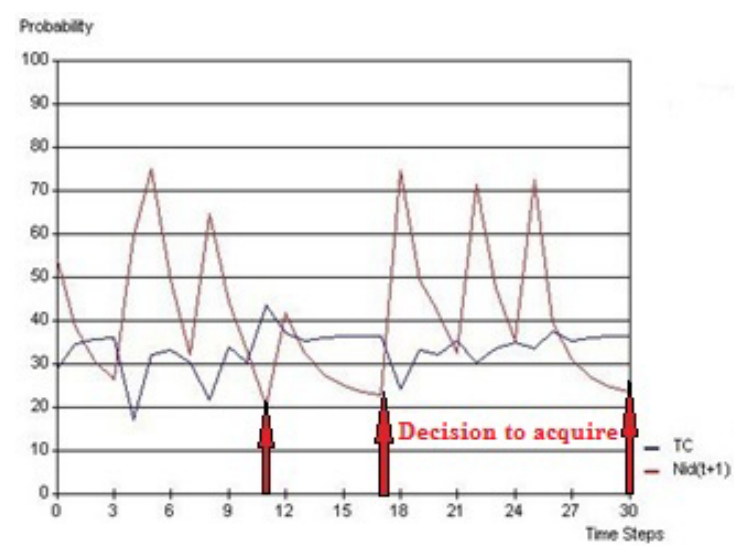

Figure 3: Reactive decisions 
Computations were performed with the help of FiCO XPREsS@ optimization tool [18] which provides a mixed integer solver and framework for constraint integer programming. Supposing a periodic review inventory with a security stock at the beginning of the period, the classical ( $\mathrm{T}$, $\mathrm{s}, \mathrm{S})$ policy is modeled in figure 2. The planning horizon was fixed to a month, and the review period is established to ten days. Results show that at the end of each review period, the number of disassembled products arrives to a minimal value and the TC to a maximal one. For the EOQ model with constant demand, a decision to acquire products to disassemble is taken whenever the inventory level reaches the reorder point.

Running simulations on the previous model, the decision to acquire is taken before the beginning of each new review period (fig. 3). In other words, starting with a periodical review inventory model, which usually provides orders at constant periods of time, we have reached to a mixed model where orders are given in accordance with the reactive decisions (i.e. in real time).

\section{Conclusions and Future Works}

An inventory model for reverse supply chains was presented. This model is deduced from the classical inventory model with constant review period and variable demand, and it encompasses decision variables structured on two decisional levels: the proactive level and the reactive level. To implement the approach, DBN was found to be an appropriate decision aid tool. Using a DBN, one can determine the optimal disassembly inventory policy dealing with stochastic aspects of the system.

Proactive decisions aim to determine the initial number of items to disassembly to fulfill the demand of parts. Reactive decisions take into consideration disassembly scenarios and the endof-life options of the disassembled parts. The model optimizes the quantity of used products to acquire so as to minimize the total inventory cost. Future work will integrate the results above in a decision support system [19]. This issue needs further investigation in real-world settings where the increased cognitive complexity of using different models (i.e. DBN, integer programming), most likely by a collective decision-maker [20], will play a major role in adopting the proposed solution.

\section{Bibliography}

[1] Gupta S. M. (2013); Reverse Supply Chains: Issues and Analysis, CRC Press, Taylor\&Francis

[2] Fleischmann M, Bloemhof-Ruwaard J. M., Dekker R. (1997); Quantitative Models for Reverse Logistics: A Review, European Journal of Operational Research, 103: 1-17.

[3] Kiesmuller G P, Scherer C W (2003); Computational issues in a stochastic finite horizon one product recovery inventory model, European Journal of Operational Research 146(3): 553-579.

[4] Inderfurth K, Langella I. M. (2006); Heuristics for solving Disassembly to order Problems with Stochastic Yields, OR Spectrum, 28 (1): 73-99

[5] Imtanavanich P, Gupta S. M. (2006); Calculating Disassembly Yields in a Multicriteria Decision Making Environment for a Disassembly to Order System, Application of Management Science, Elsevier Science, Amsterdam, 12: 109-125

[6] Bayindira Z.P., Dekkerb R, Porrasb E. (2006); Determination of recovery effort for a probabilistic recovery system under various inventory control policies, The International Journal of Management Science, 34: 571 - 584. 
[7] Inderfurth K, Langella I. M., (2008); Planning Disassembly for Remanufacture to Order Systems, Environment Conscious Manufacturing, Gupta and Lambert eds.,CRC Press, Boca Raton, Fla.

[8] Langella I. M (2007), Planning Demand Driving Disassembly for Remanufacturing, Deutscher Universitäts-Verlag.

[9] Kongar E, Gupta S M, (2009) Solving the Disassembly to Order Problem Using Linear Physical Programming, International Journal of Mathematics in Operational Research, 1(4): 504-531.

[10] Gupta, S.M., Ilgin, M. I. ( 2012), Physical Programming; A review of the state of the art, Studies in Informatics and Control, 21(4): 349-366.

[11] Ahiska S S., King R E (2010); Inventory optimization in a one product recoverable manufacturing system, International Journal of Production Economics, 124(1): 11-19.

[12] Godichaud M. (2010); Outils d'aide à la décision pour la sélection des filières de revalorisation des produits issus de la déconstruction des systemes en fin de vie. Thèse de doctorat, Université de Toulouse.

[13] Duta L., Addouche S.A. (2012); Dynamic Bayesian Network for Decision Aided Disassembly Planning, Studies in Computational Intelligence, Springer, 402: 143-154.

[14] Blumenfeld, D.E. (2008), Operations Research Calculation Handbook, CRC Press, Taylor and Francis Group.

[15] Ghorbel N., Duta L., Addouche S.A., El Mhamedi A. (2011), Decision aided tool for sustainable inventory control, The 21th International Conference on Production Research, Sttutgart, Germany.

[16] Conrady S, (2011), Introduction to Bayesian Networks, Conrady Applied Science, LLC Bayesia's North American Partner for Sales and Consulting.

[17] http://www.bayesia.com/en/products/bayesialab.php (last consulted in April 2014)

[18] FICO XPRESS Optimization Suite (http://www.fico.com/en/products/fico-xpressoptimization-suite/)

[19] Filip F.G. (2008), Decision support and control for large-scale complex systems. Annual Reviews in Control, 32(1):61-70.

[20] Zamfirescu C.B., Duta L., Iantovics B. (2010), On Investigating the Cognitive Complexity of Designing the Group Decision Process, Studies in Informatics and Control, 19 (3):263-27. 